\title{
Taller de derechos humanos en la cárcel de mujeres de Bouwer
}

Paula Leiva

Celeste Viotti

Ivana Sánchez

Micaela Reynaga

Romina Cabrera

Mayca Balaguer

Estudiantes/extensionistas de Trabajo Social,

Derecho y Psicología (UNC)

E-mail: carcelrevuelta@gmail.com 
Resumen

El Proyecto de Extensión Universitaria "Taller de Derechos Humanos en la Cárcel de Mujeres de Bouwer”, vigente desde el año 2006, es un espacio centrado en el intercambio de experiencias, conocimientos y saberes entre estudiantes de la Universidad Nacional de Córdoba y mujeres privadas de su libertad que se encuentran en el Correccional $\mathrm{N}^{\circ} 3$ de Bouwer. Durante los ocho años de existencia del proyecto han participado más de 300 mujeres privadas de su libertad en situación procesal y de condena, así como también más de 500 estudiantes y egresados/as de diversas disciplinas, incluyendo estudiantes y docentes de Derecho, Trabajo Social, Ciencias de la Información, Artes, Antropología, Psicología, Ciencias de la Educación, entre otros. El proyecto se desenvuelve a través de dos etapas: la primera es de formación, en donde docentes y estudiantes experimentados ofrecen talleres de distintas temáticas -teniendo como ejes principales y transversales Derechos Humanos y Género- para los/as futuros/ as talleristas que, en una segunda etapa, ingresarán al penal a realizar los talleres con las mujeres privadas de su libertad. Las temáticas trabajadas incluyen criminología crítica, género, educación popular, ejecución penal y justicia, entre otras.
Abstract

The university extension project "Human Rights Workshop in Bouwer's Woman's Jail”, in effect since 2006, is a space centered in the exchange of experiences and knowledge between students from Cordoba's National University, and women who are deprived of liberty in Bouwer's $\mathrm{N}^{\circ} 3$ facilities. For eight years, more than 300 women has participated, along with over 500 students and teachers from Law, Social Work, Communication Sciences, Arts, Anthropology, Psychology, Education Sciences, among others. The project develops in two stages: the first one regards the training, in which professors and advanced students offer workshops on a variety of topics - having as main axes Human Rights and Gender - to the future workshop facilitators that, on the second stage of the project, will go to the jail's facilities to replay those workshops with the woman in Bouwer. The themes cover include critical criminology, gender, popular education, penal execution, and justice among others.

\section{Key Words}

Women deprived of liberty - Human rights - University extension - Education

\section{Palabras clave}

Mujeres privadas de libertad Derechos humanos - Extensión universitaria - Educación 


\section{Introducción}

A través de la extensión, la Universidad entrelaza vínculos con la comunidad, funcionando como un actor social activo, responsable de la producción de conocimiento, no sólo puertas adentro, sino también hacia afuera. No es menor que esta institución implemente estas políticas que fortalecen el abordaje multidisciplinario (saber científico-humanístico y saber popular-social) de las distintas problemáticas que plantea la sociedad actual. Este canal -la política de extensión- genera una constante comunicación y atención a las demandas sociales; revalorizando los vínculos que se construyen en la práctica y produciendo un conocimiento que no se concibe aislado de la realidad en la que pretende incidir si no que, por sobre todo, se construye desde esa misma realidad.

En particular, el trabajo en la cárcel implica cuestionar y reflexionar sobre aspectos que pasan desapercibidos en otros espacios donde transcurre nuestra vida académica como estudiantes. Las características de dicho espacio, el tipo de vínculos y relaciones que allí observamos y de las que luego de un tiempo nos sentimos parte, nos obligan a reflexionar sobre nuestra práctica y el tipo de presencia allí; se trata de romper o ampliar los límites de estas dos instituciones: la Cárcel y la Universidad.

En los talleres se genera un "espacio sin fronteras" donde es posible el intercambio y la socialización de experiencias. Esto no sólo favorece la relación entre las mujeres privadas de su libertad, sino que logra traspasar muros y conectarse con el "afuera".

Creemos que el sentido común nos impone formas de pensar que han expuesto a las personas presas a múltiples estigmatizaciones. A través de nuestra intervención buscamos generar la deconstrucción de estos modelos estigmatizadores y problematizar la realidad carcelaria, analizando esta situación desde la Sociología, la Psicología, la Criminología, la Antropología Social, etc., para contribuir a la construcción de una sociedad más inclusiva. 
Buscamos con el siguiente artículo: - Dar a conocer el proyecto a la comunidad; • Generar vínculos con la comunidad; • Dar a conocer las condiciones del sistema penitenciario; - Hacer llegar a la comunidad las voces de las mujeres privadas de su libertad; • Romper o ampliar los límites, tanto de la institución Carcelaria como Universitaria; • Promover la sensibilización, profundización y reflexión acerca del campo de conocimiento y análisis de las prácticas en derechos humanos.

\section{Objetivos del proyecto}

En el marco de la realización de un proyecto que se continúa año tras año, y cuyos integrantes se renuevan con constancia, es primordial que el mismo cuente con una serie de objetivos generales y específicos que ordenen el quehacer cotidiano en un panorama más amplio. Sin embargo, también es importante mantener la flexibilidad para permitir que cada año se establezcan nuevos objetivos puntuales que aporten a las intencionalidades generales del proyecto, y que den lugar a que los miembros se apropien del espacio y lo re-direccionen. A continuación detallamos los objetivos generales y específicos del proyecto y los objetivos planteados para el año 2013 durante el plenario realizado en 2012:

\section{Objetivos generales:}

a) Generar un espacio de promoción jurídica y social, estableciendo vínculos de interacción con el medio libre que permitan paliar la situación de vulnerabilidad de las mujeres privadas de la libertad, a través del conocimiento de los derechos fundamentales de las personas y de los mecanismos existentes para hacerlos efectivos.

b) Difundir dentro del ámbito académico de la Universidad, la problemática carcelaria y nuestro rol como futuros operadores jurídicos y sociales interesados por dicha realidad.

c) Abordar la problemática de género, que suscita especial particularidad dentro de la entidad carcelaria y razón por la cual el trabajo de éste Proyecto se realiza en el correccional de mujeres. 


\section{Objetivos específicos:}

a) Continuar por octavo año consecutivo, conservando responsablemente dentro de la cárcel, un ámbito de discusión crítica entre estudiantes y mujeres privadas de su libertad; espacio que ha ido consolidándose profundamente, creciendo y fortaleciéndose año a año, permitiendo una amplia difusión de los derechos de los que son titulares los/las detenidos/as en particular y las personas en general, teniendo como eje básico los derechos humanos, atravesados por el género y la ejecución penal.

b) Generar un espacio de participación y de debate, que permita la interacción entre estudiantes y mujeres privadas de la libertad, logrando así actividades de capacitación, y participación colectiva desde dos saberes igualmente válidos y complementarios.

c) Fortalecer el proceso de formación de operadores jurídicos y sociales, posibilitándoles internalizar la realidad propia de la ejecución de la pena privativa de la libertad.

d) Elaborar a partir de las experiencias recogidas en la práctica un análisis reflexivo sobre la perspectiva de género en la política penitenciaria.

e) Reformular y construir un nuevo concepto de extensión universitaria que entienda a la producción de conocimientos como una práctica dialéctica, no unidireccional y la relevancia del trabajo conjunto de la Universidad Pública y el Servicio Penitenciario en las políticas públicas.

f) Participar desde nuestro rol de estudiantes extensionistas junto con el Servicio Penitenciario en la tarea del Estado en el cumplimiento de los Derechos Humanos en los contextos de encierro.

\section{Objetivos para el ciclo 2013}

Objetivos relativos a la primera etapa (formación de nuevxs integrantes del equipo):

- Generar continuidad y sustentabilidad del proyecto a través de la incorporación de nuevxs compañerxs. 
- Instalar la continuidad de la formación; la misma debe darse no sólo durante la primera etapa, sino también en paralelo con los talleres en la cárcel.

Objetivos de la segunda etapa del año (ingreso a Bouwer):

- Continuar el vínculo con las mujeres que se encuentran privadas de su libertad, y generar nuevos.

- Intensificar la extensión, a través la relación dialéctica entre talleres de formación y talleres de Bouwer, también con la difusión del trabajo.

- Funcionar como vehículos y portavoces de lo que tienen para decir las mujeres privadas de su libertad en "el afuera".

- Materializar el conocimiento construido y las voces de las mujeres de Bouwer en una publicación con formato revista.

\section{Objetivos de la comisión de Sistematización}

- Recabar toda la información.

- Producir conocimiento científico (según ejes de interés)

- Investigar sobre becas de SeCyT, Madres y/o Abuelas, CIJS

- Centralizar todos los documentos virtuales y físicos

\section{Fundamentación e historización del proyecto}

El proyecto al que pertenecemos fue presentado como propuesta de un grupo de estudiantes de la Facultad de Derecho y Ciencias Sociales, de la Universidad Nacional de Córdoba, y se enmarca dentro de un convenio firmado por dicha institución con el Correccional $\mathrm{N}^{\mathrm{o}} 3$ de Mujeres de Bouwer, que data del año 2006, bajo la premisa de extensión universitaria.

Según el Diccionario de la Real Academia Española, extensión es "acción o efecto de extender o extenderse". Podríamos decir, entonces, que la Universidad en esta tarea extiende sus puertas y muros 
hacia otro lugar, lo que nos llevaría a concluir que necesita de una acción para poder extenderse y esa acción es llevada a cabo por los distintos claustros dentro de ella, tanto los docentes-investigadoresautoridades como los/as estudiantes, formando un entrelazado de acciones para lograr extender a la Universidad. Con claridad se ha dicho que "la extensión pretende ser la función específica a través de la cual se da la relación de la Universidad Pública con la sociedad y a partir de la cual se integran sus restantes funciones -la docencia y la investigación- a la tarea de responder a las demandas de la comunidad y elaborar propuestas que permitan su desarrollo".

Desde el Proyecto de Promoción de Derechos Humanos en el Correccional $\mathrm{N}^{\circ} 3$ de Mujeres, nos proponemos ir más allá de esta conceptualización de la extensión, porque creemos que no se agota en ello, sino que la Universidad debe ser extensión en todos sus ámbitos pues se constituye como un actor social constructor, realizando entramados de conocimientos entre las distintas disciplinas (transdiciplinariedad), sujetos, objetos, realidades e instituciones de diferentes saberes, capacidades y limitaciones. Aún más, estos diversos saberes no se asientan en diferencias de jerarquías sino en variedades de formas y lugares desde donde se interroga a la realidad.

La institución universitaria debe recuperar el término "extensión" no desde extender lo propio hacia afuera, sino que debe poner en tensión lo propio con el afuera.

En este sentido, coincidimos con lo expuesto por Paulo Freire, quién plantea el término "extensión” como inadecuado a unas prácticas cuya intención fuera la verdadera educación liberadora y popular. Para este autor, la extensión como tal sólo puede consistir en la cosificación del sujeto con el que trabajamos, tomándolo como un objeto al pretender convencerlo, entregarle un conocimiento ya elaborado, e implícitamente superior al suyo, dándose en tal caso un tipo particular de invasión cultural. Propondrá Freire superar esa noción, en pos de otra como es la de "comunicación", en donde el otro con el que trabajamos sea también sujeto activo del conocimiento, y en donde se permita problematizar los saberes y colaborar en la concientización de ese sujeto. 
Nosotros vemos, por un lado, la necesidad de buscar la superación de aquella primera concepción de extensión desde el marco mismo de la (mal llamada) extensión universitaria, para trabajar desde las entrañas del sistema en su modificación; y por otro lado, nos planteamos como desafío la búsqueda constante de una posición, no tanto de concientizadores, sino más bien de promotores de la reflexión y reconstrucción de los conocimientos, de un modo conjunto e intersectorial, que siempre se oriente a la superación de las imposiciones de discursos para encaminarse hacia las prácticas dialógicas.

Sólo en la medida en que el conocimiento universitario desnaturalice y ponga en evidencia sus propias tensiones, podrá estar a la altura de las circunstancias y elaborar políticas claras para el desarrollo de una sociedad justa, democrática y equitativa, aportando a un proyecto de país acorde a ello.

Creemos fundamental como estudiantes, tener la posibilidad de encontrar otros modos de abordar el conocimiento y del futuro ejercicio de la profesión, llevando un paso más allá la eliminación de la concepción de la Universidad clasista, auto referenciada e indiferente a las demandas sociales; pues en nuestro país y particularmente en nuestra universidad -cuna de la reforma universitaria de 1918-, ciertos actores sociales le exigieron autofinanciamiento, gestión eficiente y competitividad (como si se tratase de una empresa), poniendo en riesgo su función social, desdibujándose en planes de estudio deficientes y en el vaciamiento de los recursos económicos y humanos que permitiesen llevar a cabo prácticas extensionistas con planificación seria y al alcance de estudiantes, docentes y comunidad.

Una herramienta en la construcción de la extensión es la interdisciplinariedad. Este término empleado en sentido general significa el establecimiento de relaciones entre disciplinas, mediante un procedimiento pedagógico particular. Mientras que desde nuestra posición creemos conveniente para el abordaje y trabajo de la extensión, sin importar el campo de incidencia, la utilización de la pluridisciplinariedad o multidisciplinariedad, la cual exige la yuxtaposición dialéctica y orgánica de varias disciplinas, sin ninguna tentativa de síntesis 
entre ellas. Se habla también de transdisciplinariedad cuando los saberes de los campos dispares se integran en una visión de conjunto que permite estudiar sus conexiones y relaciones de coordinación y subordinación.

Este canal, que es la política de extensión, entre las distintas disciplinas y lo social, genera una constante comunicación y atención a las demandas sociales; revalorizando los vínculos que se construyen en la práctica y produciendo un conocimiento que no se concibe aislado de la realidad en la que pretende incidir. Por ello intentamos poner la atención en el diálogo con el otro a través de nuestra metodología de talleres, quitando jerarquías al conocimiento, entendiendo que el otro no es "carente de saber" y nosotros, como representantes de la institución universitaria no somos "expertos"; por el contrario: todos/as somos poseedores/as de saberes y conocimientos diversos, y la tarea es el intercambio, nutriéndonos de todos los anteriores.

Es importante subrayar que la transdisciplinariedad no niega la importancia de los desarrollos disciplinarios, sino que los potencia a través de los diálogos y la fertilización cruzada. Es desde aquí que se puede entender con claridad la sustancia del prefijo "trans", significando a la vez un movimiento "entre", "a través de" y "más allá" de las disciplinas. Esto también promueve estrategias que tiendan a generar pasarelas entre las ciencias tecnológicas, las humanidades y las artes. Por ello mismo es que sostenemos que la problemática carcelaria puede y debe ser abordada a través de distintos campos de saber. Y es así como abrimos el Proyecto a otras unidades académicas más allá del Derecho, como ser Trabajo Social, Comunicación Social, Artes, Psicología, Humanidades en general; pues la situación de privación de libertad es compleja en todos sus aspectos y la construcción de un espacio de promoción de Derechos será, en consecuencia, abordado con la complejidad que merece para que incida en las mujeres desde su subjetividad y hasta su construcción como sujetos de derechos, destruido por el aislamiento que genera esta institución total en el sujeto que interviene.

En esta línea es importante destacar la tarea que realiza el sentido común en la construcción de ese otro: estigmatizado por su condición 
de preso, más aún se sensibiliza la situación de la mujer dentro del servicio penitenciario. Es por esto que nos proponemos en cada taller de formación con estudiantes y docentes, y en cada taller dentro de la institución carcelaria, realizar una deconstrucción de esos modelos estigmatizadores y problematizar junto con las mujeres privadas de su libertad la situación de tales, así como también poner en juego sus propios sentidos comunes respecto de otros actores o sectores sociales.

Este Proyecto, a lo largo de los años, ha logrado ser modelo de una extensión universitaria seria, poniendo en jaque la visión de "asistente social" de la universidad para con otras instituciones y siendo espacio de formación para futuros/as profesionales (tanto del Derecho, como de otras ramas sociales, técnicas y artísticas del saber), pero teniendo en claro y como punto fundamental el enfoque de derechos, que toma a los Tratados Internacionales de Derechos Humanos (DDHH) como el marco teórico y conceptual que debe guiar el diseño, implementación y ejecución de las políticas públicas de los Estados, por ser éstos garantes y responsables del cumplimiento efectivo de los derechos de los cuales gozan sus ciudadanos. Y por esto mismo nos alejamos de una práctica utilitarista o asistencialista, pues para nosotros la práctica debe ser creadora de espacios de educación no formal basadas en el diálogo. En los espacios que se crean con las mujeres privadas de su libertad, se genera una especie de "espacio sin fronteras" donde es posible el intercambio y la socialización de experiencias. Traspasando muros y conectando el adentro con el afuera; dando un paso más en las prácticas de la Universidad en la Cárcel y la Cárcel en la Universidad.

En el marco de los procesos sociales relacionados al empobrecimiento, la desafiliación, el aumento de la inequidad económica y la dificultad en el acceso a bienes sociales de primera necesidad, que se vio profundizado desde la década del ' 90 , la cárcel aparece como una respuesta política ante una coyuntura producida por un modelo socioeconómico en crisis.

La cárcel en este contexto, con sus diferentes expresiones en su desarrollo histórico, responde a las formas de articulación entre lo 
político, lo social, lo económico y lo cultural. Esto no fue ajeno a quienes encararon en su momento la ardua tarea de llevar adelante este proyecto.

Consideramos que la cárcel constituye un doble castigo para las mujeres. No sólo se castiga la comisión de un delito, sino también la transgresión de los roles asignados por la sociedad a su género, que las obliga a ser buenas madres, esposas y mujeres abnegadas. Por lo tanto, es importante remarcar que la decisión de intervenir en una cárcel de mujeres no fue casual, sino que responde a una clara necesidad de evidenciar esta situación y hacer aportes para contrarrestarla.

Por otra parte, creemos que el fin buscado por la aplicación de la pena privativa de la libertad, cuyo paradójico nombre es "resocialización", no puede nunca cumplirse en un contexto de encierro. De hecho, resulta evidente que todos los derechos humanos se ven coartados durante la permanencia en estas instituciones totalitarias.

Como estudiantes y miembros de la comunidad universitaria y de la sociedad civil, consideramos que es necesario a través de nuestras prácticas hacer visibiles estas funciones latentes de la cárcel.

Según el reconocido criminólogo italiano Alessandro Baratta, el derecho penal contemporáneo se define como "derecho penal de tratamiento", en el sentido de que se le atribuye a la pena la finalidad de reeducar y reincorporar al delincuente a la sociedad. Nuestra Constitución así lo establece en el art. 18 al decir que "Las cárceles de la Nación serán sanas y limpias, para seguridad y no para castigo de los detenidos en ellas”. Es decir, descarta la función punitiva y se enrola, junto a copiosa legislación actual, en las llamadas ideologías "re", que apuntan a la reeducación, resocialización, reinserción de los delincuentes a través del tratamiento dado en las cárceles. Sin embargo, estas funciones declaradas de la cárcel no se condicen con lo que realmente sucede. De hecho, hasta resulta poco lógico pensar que a través del encierro y el aislamiento, es decir, quitando a una persona de su contexto social y colocándola en una institución total, ésta pueda llegar a conciliarse con la sociedad cuyo orden ha roto a través del delito (Baratta, 2004). 
Teniendo en cuenta quiénes forman parte de la población carcelaria, es preciso afirmar que el derecho penal, lejano al mito de la igualdad, es el derecho desigual por excelencia. La cárcel acentúa aún más estas desigualdades, estigmatizando a quienes ocupan los últimos peldaños en la escala vertical de la sociedad, impidiendo su ascenso. Además, la sanción de ciertos comportamientos ilegales sirve para cubrir un número más amplio de comportamientos ilegales que quedan inmunes al proceso de criminalización. Se está protegiendo esta selectividad del derecho penal. La cárcel no sólo produce y reproduce esta desigualdad, sino a los sujetos pasivos de ella. Esta población marginal ejerce funciones tanto dentro como fuera de la dinámica del mercado de trabajo.

La introducción de modernas técnicas psicoterapéuticas, educativas, y de parciales transformaciones de la estructura carcelaria no ha cambiado en forma decisiva la naturaleza y la función de las instituciones carcelarias, que continúan ejerciendo efectos contrarios. Baratta plantea que la verdadera "reeducación" del condenado es aquella que transforma su razón individual y egoísta en conciencia y acción política (Baratta, 2004).

A través de nuestro proyecto, apuntamos a la realización de esa conciencia y a contrarrestar, en alguna medida, los efectos nefastos de la encarcelación. Abordamos este proyecto con la intención de crear canales de comunicación, generar un espacio libre de intercambio de palabras e ideas, construir colectivamente y reflexionar en conjunto desde las experiencias de todos/as los/as participantes para visibilizar la realidad carcelaria y hacer escuchar la voz de nuestras compañeras privadas de su libertad.

El proyecto tiene dos ejes transversales: el género y los derechos humanos. Entendemos al género como una "construcción social, cultural e histórica que configura modelos; un deber ser que se espera sea asumido como propio, que da cuenta de valores, pautas, sentires y relaciones a los que varones y mujeres deben ajustar su conducta (...) Estas construcciones socio culturales penetran en nuestra subjetividad a tal punto que llegamos a sentir y pensar de acuerdo a estos 
modelos. Se naturaliza y se vive éste deber ser como algo que siempre fue así, y que no puede ser modificado" (Lamas, 1986). El género es una manera social de tomar las pocas diferencias fácticas existentes (relativas al sexo) y subrayarlas otorgándoles una desigual distribución de poderes. Los estereotipos de los que provee la sociedad con respecto al género, se aprenden y se van reforzando en el paso por las distintas instituciones; al ser una construcción social, responden a los elementos propios de la sociedad concreta en la que nos situemos, que en el caso argentino incluyen un sistema capitalista, patriarcal, dicotómico y heteronormativo. Es por ello que el género como eje transversal del proyecto, constituye una categoría de análisis que nos permitirá no sólo visibilizar el origen de la subordinación femenina, sino también replantear "la forma de entender o visualizar cuestiones fundamentales de la organización social, económica y política” (Gonzáles y Nucci, 1999).

Con respecto al segundo eje, quienes han elaborado conceptualizaciones a lo largo de los procesos históricos, coinciden en afirmar que la principal fuente de reflexión respecto de los Derechos Humanos son las prácticas (sociales, académicas, políticas), en tanto han sido y son generadas, principalmente, por sujetos que han sufrido y sufren la flagrante violación de sus derechos. En efecto, cada acuerdo, pacto y tratado en Derechos Humanos ha surgido luego de acciones, reclamos y cuestionamientos de prácticas que degradan al hombre y la mujer y, mediante ellos, se buscó construir estándares y principios mínimos de protección y defensa. Es decir que la protección de los Derechos Humanos debe ser el objeto y a la vez el límite del poder punitivo del Estado que se plasma en las diferentes formas del control social.

Por ello asumimos que la Universidad, en tanto institución pública, tiene la responsabilidad de crear condiciones para sensibilizar, profundizar y reflexionar acerca del campo de conocimiento y análisis de las prácticas en derechos humanos, en procesos que promuevan el aprendizaje como vehículo del desarrollo de las prácticas y de la transformación de identidades, visibilizando, a su vez, las demandas de los derechos y de reconocimiento de una memoria colectiva. 
Estos dos grandes ejes son las bases de nuestros talleres que, construidos desde el paradigma de la educación popular, nos permiten intercambiar saberes, relacionando lo académico con las experiencias de todos/as sus integrantes, en un plano de igualdad.

El nombre del proyecto, "Derecho a pensar en libertad", surge bajo esa premisa: un espacio que intenta dejar de lado el encierro, los prejuicios y la desigualdad para dar lugar a la construcción colectiva de saberes y vínculos, y de esta forma recuperar la noción de semejante, afirmar, en vez de negar, la humanidad que hay en el otro.

\section{Metodología de trabajo}

El proyecto se divide en dos etapas. Durante la primera parte del año, realizamos talleres de formación dirigidos a estudiantes, comenzando con una convocatoria abierta en las facultades de la Universidad que dictan carreras de las diferentes ramas de las Ciencias sociales (Derecho, Trabajo Social, Psicología, Historia, Filosofía). Sostenemos esta convocatoria cada año desde el origen del proyecto, puesto que estamos convencidos de que el espacio será más rico si sumamos nuevos aportes y trabajamos de manera interdisciplinaria.

Además, creemos que sumar nuevos/as participantes hace a uno de nuestros principales objetivos, que es dar visibilidad a la problemática y dar a conocer lo que sucede en las cárceles. El foco está en la construcción colectiva y en la conveniencia de renovar e intercambiar desde todas las ciencias. Para llevar adelante la tarea formativa, buscamos docentes o profesionales según los ejes temáticos a trabajar, sumando la experiencia de los/as participantes del proyecto en años anteriores.

La segunda etapa del proyecto consiste en el ingreso a la cárcel por parte de los/as participantes, quienes llevan a cabo talleres con las mujeres privadas de su libertad. Los talleres abordan diferentes temáticas relacionadas a los Derechos Humanos, como ser género, trabajo, educación, entre otras. En cada taller tomamos registro de lo trabajado para luego elaborar distintos productos sobre ello fuera de 
la Cárcel, a través de diferentes intervenciones como la presentación del proyecto en diversos espacios y la confección de revistas (se publicó una en el año 2007, otra en el año 2010 y estamos trabajando en una tercera para este año).

Para organizar el trabajo del grupo de talleristas, acordamos un día de reunión semanal en donde trabajamos durante varias horas. La reunión tiene por objeto funcionar como una bisagra que articule las actividades dentro y fuera de la Cárcel. Allí socializamos las experiencias acontecidas en el Correccional durante el taller que se hubiere realizado, evaluamos críticamente la participación de los/ as talleristas y de las mujeres participantes, según los objetivos y las metas prefijadas; y nos abocamos al armado del próximo taller a dar en Bouwer.

En esta instancia, es importante destacar por qué elegimos la modalidad "taller" como la ideal para acercar la promoción de Derechos Humanos a la Cárcel. Esta opción presupone que cada sujeto es portador de conocimientos y de una historia que, desde su subjetividad, le permite significar sus propias vivencias como también los contenidos, conocimientos e ideas que colectivamente se ponen en discusión. En tal sentido, y en consecuencia, las personas que participan del proceso educativo, deben ser comprendidas como sujetos históricos y no como meros objetos de una realidad que se les impone como ajena. Partiendo de esta premisa, las reflexiones y producciones de los encuentros intentaron ser siempre colectivas, entendiendo por ello no la anulación de la subjetividad sino, por el contrario, su reconocimiento y valoración como saberes propios de las experiencias individuales, que disparan y solventan la reflexión en grupo.

Entendemos la educación desde el punto de vista de la Educación Popular, donde todos aportamos, todos decimos y somos escuchados, todos construimos y siempre en forma conjunta, sin establecer jerarquías ni diferencias; lo que implica no abocarnos a casos particulares, sino abordar las problemáticas generales que atraviesan las mujeres en situación de encierro, para que la identificación con lo trabajado sea colectiva. 
Para Paulo Freire (1980) "no hay educación popular sin toma de palabra: la palabra es poder; es creadora, transformadora de vida. Es la síntesis entre reflexión y acción (...) escucharla significa estar dispuesto a ponerla en práctica, a recrearla”. Y en este sentido creemos fundamental la participación activa de las mujeres que asisten a los talleres.

Lo lúdico es, en tal sentido, una de nuestras herramientas más importantes a la hora de problematizar los contenidos, al tiempo que posibilita una amplia y fluida comunicación no sólo mediante el lenguaje escrito u oral, sino también mediante otras formas de expresión que permiten a cada persona participar desde su propia singularidad.

Del mismo modo que con los/as estudiantes, durante nuestra primera etapa de trabajo en el año, la labor junto a la mujeres tiene en miras no sólo el acercamiento a contenidos jurídicos o científicos especialmente relacionados con la ejecución de la pena y los Derechos Humanos, sino también el abordaje de dichas temáticas desde distintos puntos de análisis crítico. De esta manera, los temas abordados en los talleres exceden al análisis del derecho positivo, y alcanzan otras cuestiones atinentes a lo social e histórico; poniendo en evidencia que partimos de una Criminología Crítica, donde convergen datos que provienen de diferentes fuentes: Sociología, Economía, Antropología, Psicología, Historia, etc., que tratan de respondernos qué es y qué pasa con el poder punitivo, con la violencia, etc.; puesto que la Cárcel misma debe ser considerada y entendida como una construcción social.

Construimos nuestro proyecto a partir del principio de que los Derechos Humanos son universales, interdependientes e indivisibles y que sólo pueden exigirse en tanto se conozcan. No puede garantizarse el desarrollo social sin el más extenso respeto de los derechos económicos, sociales, culturales, civiles y políticos. Y es así que entendemos que la Universidad debe tener un rol protagónico en la educación y promoción de derechos para contribuir a la neutralización de la vulnerabilidad de los grupos sociales marginados.

Para realizar un seguimiento del Proyecto llevamos adelante, en cada taller, la tarea de registro que conlleva la labor de apuntar todo 
lo que va aconteciendo durante el taller, no sólo lo que se habla, sino todo lo que se dice en las miradas, los silencios, la posición de los cuerpos, etc. Los registros nos permiten hacer luego un análisis más acabado de todo lo sucedido. Este seguimiento es acompañado de la socialización, experiencia que lleva a volcar dentro del colectivo el aprendizaje individual de cada tallerista, aportando a la construcción de un saber de todos/as los/as participantes.

Finalmente, la instancia de evaluación del Proyecto, es realizada en jornadas de plenario, de las cuales participamos todos/as los/as talleristas con el objetivo de re-discutir las bases que sentamos durante el desarrollo del trabajo anual, para proyectar la continuación del Proyecto.

Planteada esta metodología, consideramos que el trabajo extensionista se desarrolla constantemente: articulando la institución carcelaria junto a la universitaria, pero también articulando a las distintas unidades académicas y a los claustros docente y estudiantil entre sí; como también reconociendo el carácter social propio de la extensión, a través de un análisis criminológico crítico y por medio de la educación no formal, de planteo en talleres de carácter popular.

\section{Conclusión}

Del análisis del desempeño durante el año 2013, y la contrastación con los objetivos que nos hubiéramos planteado para el mismo, se desprenden diversas conclusiones.

Consideramos que se logró ampliar el grupo y darle continuidad al proyecto a través de la incorporación de nuevos/as compañeros/as, si bien esto también generó nuevos desafíos a la hora de promover la coordinación y comunicación dentro de un grupo más numeroso. También se continuó el vínculo con las mujeres privadas de su libertad.

Por otra parte, consideramos necesario insistir en los procesos de formación continua, ya que siguen resultando dos etapas muy desvinculadas la de formación respecto a la de ingreso al penal. Para ello 
se planteó aprovechar más las instancias de foros y capacitaciones propuestas por terceros, y así continuar la formación durante la segunda mitad del año, sin sobrecargar nuestros horarios teniendo que idear talleres para tales fines. También creemos que fue insuficiente el trabajo como portavoces de los discursos de las mujeres privadas de su libertad, ya que no concluimos con el proceso de producción de la revista en los tiempos esperados; con lo cual reforzamos nuestro compromiso con la realización de la misma, y buscamos otros espacios de difusión para aportar a esa tarea, como la escritura de textos académicos y las entrevistas en programas de radio que permitan propagar la experiencia y la realidad de las mujeres en Bouwer.

En base al camino recorrido, consideramos importante prevenir sobre las dificultades que hemos encontrado en el proceso, y comentar cuáles son los objetivos que nos proponemos a futuro.

En cuanto a lo primero, ha habido diversidad de obstáculos impuestos por el funcionamiento del sistema penitenciario a la hora de ingresar a realizar los talleres, que en general terminan sorteándose, pero que constituyen un modo de disuasión que la institución nos impone.

A su vez, al ser un grupo numeroso de participantes, siempre es un desafío organizar las reuniones y mantener una comunicación fluida. Al principio, con las nuevas incorporaciones, tuvimos situaciones de desorganización, ya que todos contábamos con medios de comunicación diferentes. Hemos podido solucionar estos problemas estableciendo una única vía de comunicación y de esta manera podemos mantenernos al tanto mutuamente.

También en algunas oportunidades nos vimos afectados/as por situaciones de tensión que ocurrieron al interior de los talleres, en donde muchas veces no hallamos respuestas ya que nos tomaban por sorpresa. En distintas oportunidades las participantes de los talleres se encuentran desbordadas, sensibilizadas por alguna situación particular que ha ocurrido dentro del penal, y eso impide la concreción de los objetivos específicos que tenemos para cada encuentro. En estos casos, si bien nos encontrábamos desprevenidos, logramos canalizar 
la tensión y trabajar sobre lo que había sucedido a través de alguna técnica o metodología grupal. Por esto mismo es que creemos fundamental la formación previa antes del ingreso al penal.

En lo relativo a nuestros objetivos a futuro, además de seguir sosteniendo el espacio (tanto la capacitación con nuevos/as compañeros/ as, como los talleres en el sistema penitenciario), durante el plenario en el que analizamos los resultados de nuestro desempeño durante el 2013, surge la necesidad de:

- Comunicar y difundir más el proyecto, para que otros/as se sumen a formar parte del mismo y para generar una conciencia y visibilización de la situación de las mujeres privadas de su libertad, y de un sistema penal que no cumple con los objetivos que se propone.

- Ser vehículos de las voces de las mujeres que se encuentran en Bouwer, transmitiendo sus experiencias fuera de los muros de la cárcel.

- Profundizar nuestra capacitación como equipo en las temáticas que trabajamos, convirtiéndola en una actividad constante y no sólo delimitada a cierto período del año.

- Sistematizar las experiencias para recuperarlas y analizarlas con facilidad a la hora de tomar decisiones.

- Recuperar la palabra para construir diferentes soportes sociales.

- Abrir a los sujetos a nuevas posibilidades de acceso en la vida social y cultural, generando un vínculo de redes entre ellas, nosotros/ as y la sociedad.

Finalmente, consideramos que la tarea de promover Derechos Humanos en una cárcel no es sencilla. Puede decirse que es una contradicción. ¿Quién más que esas mujeres privadas de la libertad saben que en la cárcel se violan sistemáticamente los Derechos Humanos? Es en este punto en el que ponemos atención al definir las líneas de trabajo. La idea de promover derechos es generar una profundización en el fortalecimiento de la concepción de las personas privadas de la libertad como sujetos de derechos no sólo dentro de la cárcel sino fuera de ella. 
Todos los años, cuando comenzamos los talleres surgen las mismas preguntas: ¿Dónde están los Derechos Humanos? ¿Dónde está la justicia? Las afirmaciones: "acá hay justicia para unos pocos, no para todas"; "Quiero saber qué es la justicia, quiero que me la expliquen" (registros de los talleres, 2013) son las más recurrentes al hablar de cualquier temática propuesta. Por esta razón es necesario que la Universidad Pública se constituya como un actor social activo que incida en esa realidad e intente transformarla.

Al entender de Garland, el castigo es "una encarnación práctica de algunos de los temas simbólicos, constelaciones de significados y formas específicas de sentir que constituyen a la cultura en general", dónde las instituciones penales construyen y difunden significados culturales. En su práctica rutinaria la penalidad difunde nociones definidas de lo que debe ser una persona, qué tipos de personas hay y como deben ser entendidas dichas personas y sus subjetividades, las políticas del castigo, los discursos y las instituciones sostienen conceptos específicos de subjetividad y autorizan formas de identidad de individuos. Partiendo de estas consideraciones, nos proponemos junto con las mujeres, generar un espacio de libertad de expresión, donde la palabra se constituye como una herramienta de empoderamiento en un espacio en que la subjetividad es atravesada por un sistema de control permanente sobre el cuerpo: "Podrán quitarme la libertad, pero nunca podrán quitarme mi persona" (registros de los talleres, 2012).

Las mujeres nos expresan la importancia de estos espacios, en donde se valora la palabra y las ideas de cada uno/a. Siempre teniendo como norte que "Ante cualquier circunstancia somos personas. Exigimos respeto y dignidad" (registros de los talleres, 2012). 


\section{Referencias bibliográficas}

BARATTA, A. Criminología y Sistema Penal, In memoriam. Buenos Aires, B de F Editora, 2004.

BARRIENTOS, M. "Compendio bibliográfico - Asignatura UNC”. Córdoba, mímeo, 2008 .

CELS (Centro de estudios legales y sociales), Ministerio Público de la Defensa de la Nación-Procuración Penitenciaria de la Nación. Mujeres en Prisión. Los alcances del castigo. Buenos Aires, Siglo XXI editores, 2011.

DAROQUI, A. Veinte años de la Carrera de Sociología en el programa UBA XXII Universidad en las cárceles. Buenos Aires, Editorial Yael, 2009.

FOCAULT, M. "La sociedad punitiva”, en: FOUCAULT, M. La vida de los hombres infames. Buenos Aires, Ed. Caronte, 1996.

FOCAULT M. Vigilar y Castigar. Nacimiento de la Prisión. Buenos Aires, Siglo XXI Editores, 2009.

FREIRE, P. ¿Extensión o comunicación? La concientización en el medio rural. Montevideo, Siglo XXI Editores, 1973.

FREIRE P. Pedagogía del Oprimido. Buenos Aires, Biblioteca Clásica S. XXI, 2010.

GARLAND, D. Castigo y sociedad moderna. México, Siglo XXI editores, 1999.

GOFFMAN, E. Internados. Buenos Aires, Siglo XXI Editores, 2008.

GONZALES, C. y NUCCI, N. "Necesidades y satisfactores en el análisis del espacio familiar. Córdoba: Informe de Investigación con Aval de la SECyT de la UNC para el programa de Incentivos”. Córdoba, mímeo, 1999.

LAMAS, M. La antropología feminista y la categoría "género". México, Nueva antropología, 1986.

MERCADO, L. Juego y Recreación en Educación. Córdoba, Editorial Brujas, 2011.

UNIVERSIDAD NACIONAL DE CÓRDOBA. Secretaría de Extensión.

Pronunciamiento sobre la función de la extensión en la Universidad Pública. II Foro de Extensión Universitaria. Córdoba, 2013. [En línea: 10/02/2014]. Disponible en: http:// www.unc.edu.ar/extension-unc/gestion/consejo-asesor/foros-de-extension/tercer-foro-deextension/unc_seu_pronunciamiento_iii_foro_extension.pdf/at_download/file

Recepción: 14/03/2014

Aceptación: 19/04/2014 